\title{
Methicillin-Resistant Staphylococcus aureus Laryngitis: A Report of Two Cases with Different Clinical Presentations
}

\author{
Marcelo B. Antunes Evan R. Ransom Kevin P. Leahy \\ Department of Otorhinolaryngology, Head and Neck Surgery, University of Pennsylvania, Philadelphia, Pa., USA
}

\author{
Key Words \\ Methicillin-resistant Staphylococcus aureus • Laryngitis • \\ Airway obstruction
}

\begin{abstract}
Background: Methicillin-resistant Staphylococcus aureus (MRSA) infection has been described in multiple areas of the head and neck. Recently, otolaryngologists have recognized MRSA infection in the glottis. We describe 2 cases of MRSA laryngitis with divergent clinical presentations: acute airway obstruction and recalcitrant hoarseness. Methods: Report of 2 cases and review of the literature. Results: In the first case, a 44-year-old woman presented with near aphonia despite maximal medical therapy. Examination showed diffuse erythema and edema of the endolarynx with yellowish plaques lining the glottis and supraglottis. Complete resolution was achieved with long-term trimethoprim-sulfamethoxazole. In the second case, a 54-year-old woman presented with recent-onset hoarseness with rapid progression to respiratory distress and biphasic stridor. Endoscopy revealed exuberant granulation tissue in the glottis with a narrowed airway. Treatment required prolonged courses of antibiotics and steroids. Diagnosis in both cases was confirmed with biopsies taken during direct laryngoscopy. Conclusions: MRSA treatment is a growing part of otolaryngologic practice and should be included in the differential diagnosis of hoarseness and stridor.

Copyright $\odot 2012$ S. Karger AG, Basel
\end{abstract}

\section{Introduction}

Methicillin-resistant Staphylococcus aureus (MRSA) was initially described in the 1960s [1]. Since then, the rate of hospital and community-acquired infection has been rising dramatically. The incidence of MRSA in hospitals increased from $2.4 \%$ of the S. aureus infections in 1975 to $43 \%$ in 2002 [2]. In addition, the incidence of communityacquired MRSA infections tripled from 1999 to 2003 [3].

The incidence of MRSA infections in the head and neck has equally been increasing concomitantly over the past three decades. MRSA has been recovered from a variety of clinically evident infections as well as nasal mucosa of normal individuals [4]. It has been described in epiglottitis [5], deep neck infections $[6,7]$, otitis media and externa, chronic tonsillitis [8] and in the glottis [9, 10]. To our knowledge, these are the only 2 reports of MRSA laryngitis published in the English language literature. We describe 2 cases of laryngitis caused by MRSA with different clinical presentation: the first with chronic laryngitis unresponsive to medical therapy and the second with subacute airway obstruction.

\section{Case Reports}

Case 1

A 44-year-old woman with a medical history significant for HIV and hepatitis C infection, gastroesophageal reflux and tobacco use, presented to the office with a 9-month history of hoarse-

\section{KARGER}

Fax +41613061234

E-Mail karger@karger.ch

www.karger.com
(C) 2012 S. Karger AG, Basel

$0301-1569 / 12 / 0743-0146 \$ 38.00 / 0$

Accessible online at:

www.karger.com/orl
Marcelo Antunes, MD, Department of Otorhinolaryngology

Head and Neck Surgery, Hospital of the University of Pennsylvania

3400 Spruce Street, 5 th floor Silverstein Building

Philadelphia, PA 19104 (USA)

Tel. +1 215662 2137, E-Mail marcelo.antunes@uphs.upenn.edu 


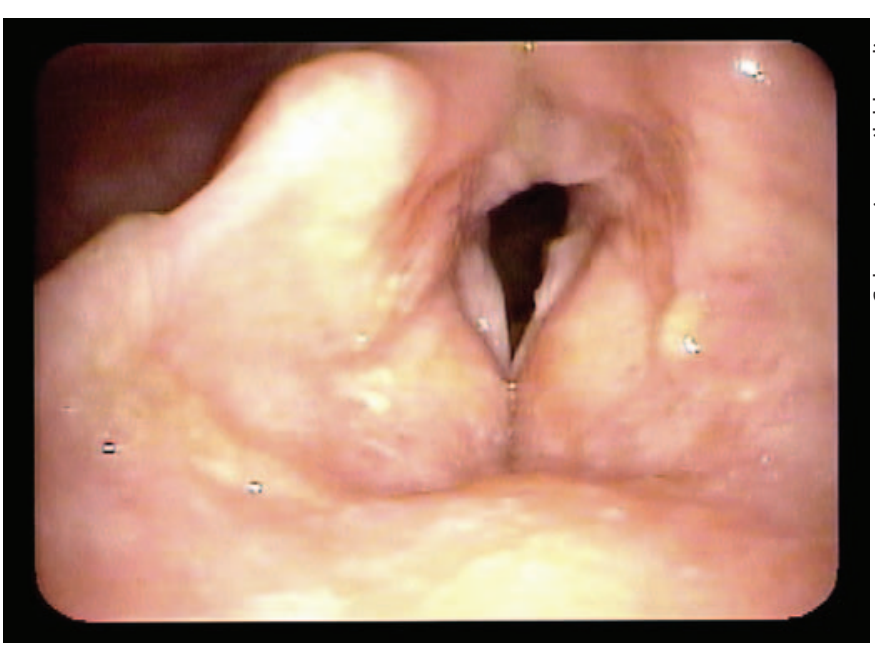

Fig. 1. Pretreatment endoscopic view with left true vocal fold mucosal irregularity.

ness and globus sensation that progressed to neck pain over the left side of her larynx. She did not have associated dysphagia or respiratory distress. She had failed multiple courses of antibiotics that included cephalexin and amoxicillin-clavulanate. The patient had been an intravenous drug abuser and was imprisoned briefly in the year prior to having symptoms. On examination, she was found to have mild bilateral true vocal fold erythema with mucosal irregularity on the left side (fig. 1). The patient failed to improve with a course of proton pump inhibitors and moxifloxacin, and the decision was made to perform a direct laryngoscopy with biopsy and cultures. Microscopic inspection showed diffuse thickening of the true vocal fold mucosa without a discrete mucosal lesion and presence of exudate on the glottis and supraglottis. Gram stain and cultures confirmed MRSA. The patient was then treated with trimethoprim-sulfamethoxazole (Bactrim-DS) in addition to a daily proton pump inhibitor. She completed a 6-week course with nearcomplete resolution of her symptoms.

\section{Case 2}

A 54-year-old woman with a medical history significant for asthma, gastroesophageal reflux, sarcoidosis and tobacco use presented to the office with a 1-month history of worsening respiratory distress, hoarseness and stridor that had failed to improve with a course of steroids and amoxicillin-clavulanate. She reported a history of a severe upper respiratory infection about 2 months prior to her visit. On fiberoptic examination, she was found to have laryngeal edema with granulation tissue and fibrinous exudate over the true vocal cords and diffuse erythema and edema over the posterior glottis and postcricoid region (fig. 2). The patient was taken urgently for a direct laryngoscopy with biopsy and cultures. The granulation tissue was debulked with a microdebrider. Due to concerns about airway edema, the patient was left intubated overnight and extubated the following morning. She was placed on steroids and broad-spectrum antibiotics pending culture and biopsy results.

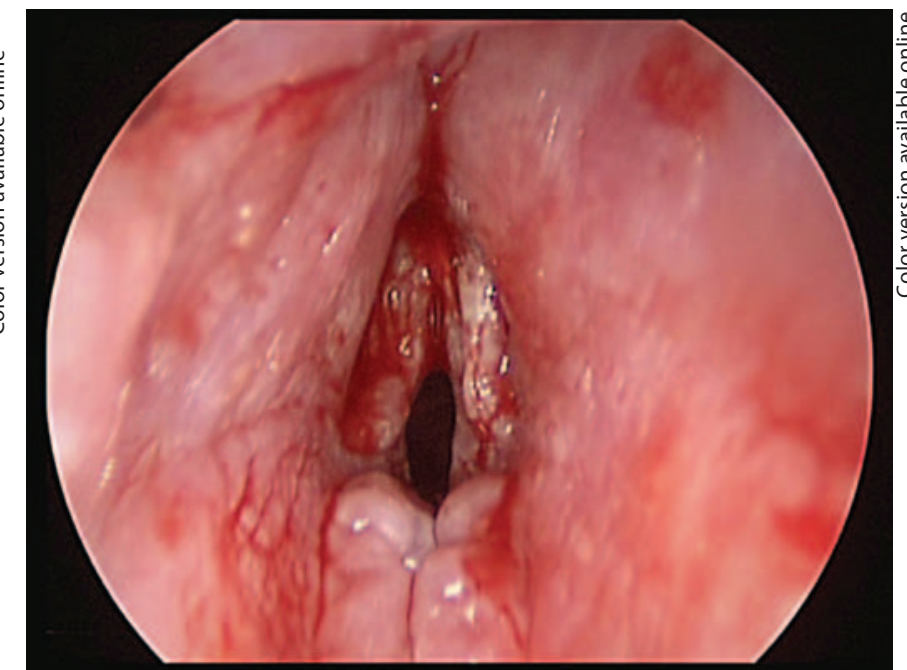

Fig. 2. Pretreatment endoscopic view with exuberant granulation tissue in the vocal folds.

The histopathologic analysis revealed granulation tissue with acute inflammation as well as Gram-positive cocci in clusters. Cultures confirmed MRSA, which was sensitive to vancomycin, sulfamethoxazole, rifampin and gentamicin. The patient was started on Bactrim-DS, and the steroids were tapered. One-week follow-up in the office revealed the presence of persistent laryngeal inflammation, yellow crusting and white patches on endoscopy. She was then additionally given fluconazole for presumed candidiasis, as well as rifampin, alongside Bactrim-DS. On her second follow-up visit, she was feeling better with improvement in her voice and shortness of breath. Repeat endoscopy showed decreased laryngeal inflammation. A total of 6 weeks of BactrimDS and 4 weeks of rifampin was prescribed. At 4 months of follow-up, she demonstrated significant improvement in voice quality and shortness of breath (fig. 3).

\section{Discussion}

Infectious laryngitis can be acute, subacute or chronic. The acute and subacute forms are usually abrupt, developing over a period of less than 3 weeks, while chronic laryngitis may develop much more gradually. Chronic laryngitis can be caused by viral, fungal or bacterial infection. In the latter, the disease process usually starts after inhalation of bacteria from an infected or colonized individual. MRSA infections are generally more virulent than those of methicillin-sensitive $S$. aureus, with a higher mortality rate [11].

To the authors knowledge there are only 2 reports of laryngitis caused by MRSA in the English language lit- 


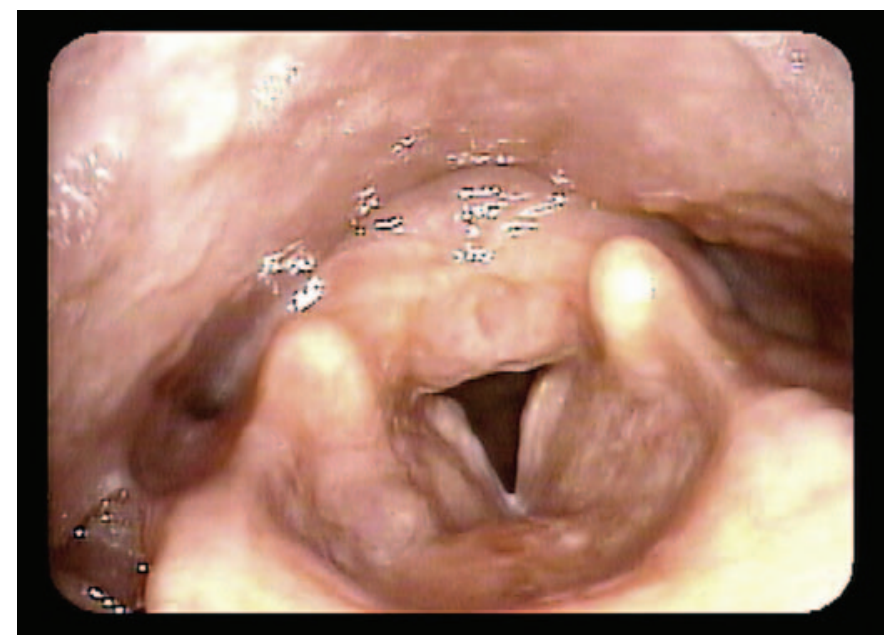

Fig. 3. Posttreatment endoscopic view with significant improvement.

erature: 1 in a child and 1 in an adult $[9,10]$. In the first, an 11-month-old child was found to have MRSA, influenza B and Candida growth from laryngeal plaques. The second describes a case of MRSA infection following an episode of upper respiratory tract infection in a patient with immunodeficiency that failed to improve with standard maximal medical therapy.

Similarly to the latter case, our first case also had chronic symptoms and had failed multiple treatment modalities. Our patient had risk factors for MRSA infection being HIV positive, an intravenous drug abuser and a former prison inmate, which raised the suspicion. How- ever, one of the most important factors to take into consideration is the lack of improvement with standard or first-line treatment. In our second case, similar to the previously published case, the patient also had a previous episode of respiratory infection. This happened during the H1N1 outbreak, and even though the patient did have this virus formally diagnosed, the severity of the symptoms described by the patient was consistent with that viral infection. This patient also had multiple factors that would make the larynx chronically inflamed such as gastroesophageal reflux and tobacco smoking. All of these factors could increase the baseline inflammatory level in the larynx that would be further aggravated by the staphylococcal exposure. This could possibly explain why the infectious insult resulted in such an exuberant inflammatory reaction and led to airway distress. MRSA laryngitis presenting as acute respiratory distress has not been previously reported.

\section{Conclusions}

MRSA infections are becoming more prevalent in the head and neck. Laryngitis caused by MRSA is still a rare entity. Nonetheless, with the increased presence of this strain in the community, this should be included in the differential diagnosis of acute or subacute inflammatory processes of the larynx. The 2 cases reported here also illustrate the clinical situation that when patients fail to improve with standard therapy, further diagnostic modalities, including direct laryngoscopy with biopsy, might be necessary to make a diagnosis.

\section{References}

$>1$ Enright MC, Robinson DA, Randle G, Feil EJ, Grundmann H, Spratt BG: The evolutionary history of methicillin-resistant Staphylococcus aureus (MRSA). Proc Natl Acad Sci USA 2002;99:7687-7692.

- 2 Collins M, Tami TA: Methicillin-resistant Staphylococcus aureus (MRSA) in the practice of otolaryngology - an emerging community acquired organism? Curr Opin Otolaryngol Head Neck Surg 2003;11:179-183.

-3 Bothwell NE, Shvidler J, Cable BB: Acute rise in methicillin-resistant Staphylococcus aureus infections in a coastal community. Otolaryngol Head Neck Surg 2007;137:942-946.

-4 Kuehnert MJ, Kruszon-Moran D, Hill HA, McQuillan G, McAllister SK, Fosheim G, McDougal LK, Chaitram J, Jensen B, Fridkin
SK, Killgore G, Tenover FC: Prevalence of Staphylococcus aureus nasal colonization in the United States, 2001-2002. J Infect Dis 2006;193:172-179.

-5 Freeman L, Wolford R: Acute epiglottitis caused by methicillin-resistant Staphylococcus aureus in adults. Clin Infect Dis 1998;26: 1240-1241.

-6 Falup-Pecurariu O, Leibovitz E, Pascu C, Falup-Pecurariu C: Bacteremic methicillin-resistant Staphylococcus aureus deep neck abscess in a newborn - case report and review of literature. Int J Pediatr Otorhinolaryngol 2009;73:1824-1827.

-7 Chern SH, Wei CP, Hsieh RL, Wang JL: Methicillin-resistant Staphylococcus aureus retropharyngeal abscess complicated by a cervical spinal subdural empyema. J Clin Neurosci 2009;16:144-146.

8 Brook I, Foote PA: Isolation of methicillin resistant Staphylococcus aureus from the surface and core of tonsils in children. Int J Pediatr Otorhinolaryngol 2006;70:2099-2102.

>9 Somenek M, Le M, Walner DL: Membranous laryngitis in a child. Int J Pediatr Otorhinolaryngol 2010;74:704-706.

10 Liakos T, Kaye K, Rubin AD: Methicillin-resistant Staphylococcus aureus laryngitis. Ann Otol Rhinol Laryngol 2010;119:590593.

11 Kaye KS, Engemann JJ, Mozaffari E, Carmeli Y: Reference group choice and antibiotic resistance outcomes. Emerg Infect Dis 2004; 10:1125-1128. 